Journal of Zhejiang University-SCIENCE B (Biomedicine \& Biotechnology) ISSN 1673-1581 (Print); ISSN 1862-1783 (Online)

www.zju.edu.cn/jzus; www.springerlink.com

E-mail: jzus@zju.edu.cn

\title{
Review:
}

\section{Antibiotic resistance mechanisms of Myroides sp."}

\author{
Shao-hua $\mathrm{HU}^{\S 1}$, Shu-xing YUAN ${ }^{\S 2}$, Hai QU ${ }^{3}$, Tao JIANG ${ }^{1}$, \\ Ya-jun ZHOU ${ }^{1}$, Ming-xi WANG ${ }^{\dagger 1,4}$, De-song $\mathrm{MING}^{\dagger+5}$ \\ ( ${ }^{1}$ Yun Leung Laboratory for Molecular Diagnostics, School of Biomedical Sciences and Institute of Molecular Medicine, Huaqiao University / \\ Engineering Research Center of Molecular Medicine, Ministry of Education, Xiamen 361021, China) \\ ( ${ }^{2}$ Department of Neurosurgery, Linyi People's Hospital, Linyi 276000, China) \\ $\left({ }^{3}\right.$ Linyi Health School of Shandong Province, Linyi 276000, China) \\ ( ${ }^{4}$ Institute of Nanomedicine, Department of Medical Laboratory, Weifang Medical College, Weifang 261053, China) \\ ( ${ }^{5}$ Department of Clinical Laboratory, Quanzhou First Hospital Affiliated to Fujian Medical University, Quanzhou 362000, China) \\ †E-mail: mxwang@hqu.edu.cn; mds6430@126.com \\ Received Mar. 19, 2015; Revision accepted Nov. 30, 2015; Crosschecked Feb. 15, 2016
}

\begin{abstract}
Bacteria of the genus Myroides (Myroides spp.) are rare opportunistic pathogens. Myroides sp. infections have been reported mainly in China. Myroides sp. is highly resistant to most available antibiotics, but the resistance mechanisms are not fully elucidated. Current strain identification methods based on biochemical traits are unable to identify strains accurately at the species level. While $16 \mathrm{~S}$ ribosomal RNA (rRNA) gene sequencing can accurately achieve this, it fails to give information on the status and mechanisms of antibiotic resistance, because the 16S rRNA sequence contains no information on resistance genes, resistance islands or enzymes. We hypothesized that obtaining the whole genome sequence of Myroides sp., using next generation sequencing methods, would help to clarify the mechanisms of pathogenesis and antibiotic resistance, and guide antibiotic selection to treat Myroides sp. infections. As Myroides sp. can survive in hospitals and the environment, there is a risk of nosocomial infections and pandemics. For better management of Myroides sp. infections, it is imperative to apply next generation sequencing technologies to clarify the antibiotic resistance mechanisms in these bacteria.
\end{abstract}

Key words: Myroides sp., Antibiotic resistance, Identification methods, 16 S ribosomal RNA gene sequencing, Next generation sequencing

http://dx.doi.org/10.1631/jzus.B1500068

CLC number: R378

\section{Introduction}

The genus Myroides (Myroides spp.) comprises yellow-pigmented, non-motile, Gram-negative, rodlike bacteria (Holmes et al., 1977; Cho et al., 2011)

\footnotetext{
Corresponding authors

$\S$ The two authors contributed equally to this work

* Project supported by the Huaqiao University Graduate Student Scientific Research Innovation Ability Cultivation Plan Projects, the Major Program of Department of Science and Technology of Fujian Province (No. 2012Y4009), the Science and Technology Planning Project of Xiamen (No. 3502Z20123036), the Xiamen Southern Oceanographic Center (No. 14GYY008NF08), the Construction Project for Yun Leung Laboratory for Molecular Diagnostics (No. 14X30127), the Technology Planning Projects of Quanzhou Social Development Fields (No. 2014Z24), and the Major Support Research Project of National Key Colleges Construction of Quanzhou Medical College (No. 2013A13), China

(i) ORCID: Ming-xi WANG, http://orcid.org/0000-0002-8093-0384

(C) Zhejiang University and Springer-Verlag Berlin Heidelberg 2016
} that release a fruity odor during growth (Holmes et al., 1977). The first strain, Stutzer, of the genus Myroides was isolated from the stools of patients with intestinal infections (Holmes et al., 1977) and was assigned the species name Flavobacterium odoratum (Stutzer and Kwaschnina, 1929). For easier clinical recognition, the bacteriological features, pigmentation, biochemical characteristics, and antimicrobial profiles of 10 isolates were examined (Holmes et al., 1977). Myroides spp. were found to be non-fermentative 
organisms resistant to many antibiotics (Holmes et al., 1977). In 1996, after extensive polyphasic taxonomic analysis of 19 strains of $F$. odoratum, the genus $M y$ roides was established and included two species, $M$. odoratus and M. odoratimimus (Vancanneyt et al., 1996). Later, more strains were isolated from forest soil (strain TH-19(T), named M. xuanwuensis sp. nov. (Zhang et al., 2014)), seawater (strain JS-08(T), named M. marinus sp. nov. (Cho et al., 2011), strain SM1(T), named M. pelagicus sp. nov. (Yoon et al., 2006)), deep-sea sediment (strain D25T (Zhang et al., 2008)), human saliva (strain MY15T, named $M$. phaeus sp. nov. (Yan et al., 2012)), as well as strains from urine, sputum, surgical exudate (Andreoni, 1986), and patients' matter (Table 1). Thus, Myroides spp. are widely distributed in nature (Mammeri et al., 2002; Ktari et al., 2012; Suganthi et al., 2013; Ravindran et al., 2015).

Myroides sp. is a rare opportunistic pathogen (Schröttner et al., 2014). Nevertheless, management of Myroides sp. infection is troublesome due to its high resistance to most antibiotics (as summarized in Table 1). For accurate strain identification of $M y$ roides sp., current diagnostic methods, such as the Vitek Jr. system (Vitek Systems, bioMerieux) (Spanik et al., 1998), are based on bacteriological and biochemical characteristics, and can determine $M y$ roides $\mathrm{sp}$. at the species level in most cases. However, they and 16S ribosomal RNA gene sequencing (16S rRNA sequencing), a standardized bacterial strain identification method (Yoon et al., 2006; Zhang X.Y. et al., 2008; Zhang Z.D. et al., 2014) still not widely applied in Chinese hospitals, fail to provide any information on the status and mechanisms of antibiotic resistance in Myroides sp. Whole genome sequencing technologies could address these questions, and should be applied to Myroides sp. promptly.

\section{Antibiotic resistance status of clinical Myroides sp. infections}

Myroides sp. infections are rare. By searching the PubMed database of English literature using "Myroides" or "Flavobacterium odoratum" as key words, only a few reports could be found. In immunocompetent people, primary infections by Myroides sp. have been rarely reported, such as a case of $M$. odoratimimus cellulitis resulting from a pig bite in an immunocompetent child (Maraki et al., 2012). However, secondary infections can frequently arise when human immunity is impaired, such as post catheterization (Holmes et al., 1977; Spanik et al., 1998), in patients with cancer (Holmes et al., 1977; Spanik et al., 1998; Song, 2005) or diabetes mellitus (Yang and Wang, 2001), and in neonates (Wang and Su, 1992; Zhang and Zhang, 1996; Zhao, 2000). Myroides sp. can cause soft tissue infection (Benedetti et al., 2011), cellulitis (Bachmeyer et al., 2007), necrotizing fasciitis (Crum-Cianflone et al., 2014), ventriculitis (Macfarlane et al., 1985), and urinary tract infections (Yağci et al., 2000). M. odoratimimus even caused an outbreak of urinary tract infection in a hospital (Ktari et al., 2012).

By using the same key words to search the China National Knowledge Infrastructure (CNKI) database, we found that most reports of Myroides or F. odoratum infections contained a single case (Table 1). Two papers reported 23 (Table 2) and 11 strains (Table 3), respectively.

From Tables 1, 2, and 3, we conclude that $M y$ roides spp. are resistant to broad antibiotics, and that their extensive antibiotic resistance has resulted in treatment failure and fatalities. We observed a case in July 2009 in which a patient presented with a post-injury urinary tract infection caused by $M$. odoratimimus strain PR63039 (Table 1, our case). Using antibiotic sensitivity testing (AST), the strain was found to be resistant to ampicillin, amoxicillin, clavulanate, amikacin, aztreonam, chloramphenicol, cephalosporin, imipenem, gentamycin, levofloxacin, meropenem, shubatan, sulfamethoxazole, tetracycline, ciprofloxacin, and tazobactam. Even though many antibiotics, such as cefazolin oxime, amikacin, tetracycline, moxifloxacin, ciprofloxacin, and nitrofurantoin, were administered to the patient for $47 \mathrm{~d}$, the infection was not cured.

The reports analyzed in Table 1 reveal that the antibiotic resistance of Myroides sp. varies among strains isolated from different sources. For example, a strain from a patient suffering from a hydatid cyst of the liver was sensitive to norfloxacin (An, 1992), but another strain isolated from pulmonary infection patient was reported to be resistant to norfloxacin (Liu and $\mathrm{He}, 2001)$. Two strains isolated from patients with cellulitis and a leg amputation, respectively (Hu et al., 2013; Crum-Cianflone et al., 2014) were resistant to 
ciprofloxacin, while another two isolated from trauma and septicemia patients, respectively, were sensitive to ciprofloxacin (Geng et al., 2000; Sun and Zhang, 2006).

Why is there so much variation in the antibiotic resistance profiles of Myroides sp. strains isolated from different sources? In our opinion, the subtypes and genotypes of Myroides sp. might have a great influence on their sensitivity to certain antibiotics. Therefore, it is imperative to obtain accurate information on strain subtype and genotype.

Table 1 Summary of reported infections by Myroides sp.

\begin{tabular}{|c|c|c|c|c|c|c|c|}
\hline $\begin{array}{l}\text { Patient } \\
\text { No. }\end{array}$ & $\begin{array}{l}\text { Age } \\
\text { (year)/ } \\
\text { gender }\end{array}$ & $\begin{array}{l}\text { Underlying } \\
\text { diseases or } \\
\text { reasons }\end{array}$ & Site of isolation & Antibiotic resistance status & Treatment strategy & Outcome & Reference \\
\hline 1 & $87 / \mathrm{M}$ & $\begin{array}{l}\text { Trauma, } \\
\text { old age }\end{array}$ & Wound & $\begin{array}{l}\text { Resistant to all antibiotics except } \\
\text { ciprofloxacin. Sensitive to } \\
\text { trimethoprim-sulfamethoxazole }\end{array}$ & iv ciprofloxad & Favorable & $\begin{array}{l}\text { Sun and } \\
\text { Zhang, } \\
2006\end{array}$ \\
\hline 2 & $34 / F$ & $\begin{array}{l}\text { Hydatid cyst } \\
\text { of liver }\end{array}$ & Drainage & $\begin{array}{l}\text { Resistant to neomycin, streptomycin, } \\
\text { gentamicin, ampicillin, tobramycin. } \\
\text { Sensitive to norfloxacin }\end{array}$ & Norfloxacin & Favorable & An, 1992 \\
\hline 3 & $2 / \mathrm{M}$ & Young age & $\mathrm{CSF}$ & $\begin{array}{l}\text { Resistant to cefazolin, penicillin, } \\
\text { chloramphenicol. Sensitive to ampicillin, } \\
\text { polymyxin, kanamycin, erythromycin, } \\
\text { neomycin }\end{array}$ & ND & ND & $\begin{array}{l}\text { Shi and } \\
\text { Zhou, } \\
1993\end{array}$ \\
\hline 4 & $71 / \mathrm{M}$ & $\begin{array}{l}\text { Chronic } \\
\text { bronchitis, } \\
\text { old age }\end{array}$ & Sputum & $\begin{array}{l}\text { Resistant to meropenem, imipenem, } \\
\text { ampicillin, cefradine, tobramycin, } \\
\text { cephalothin, amoxicillin-clavulanic acid, } \\
\text { ampicillin-shubatan, ceftriaxone, } \\
\text { gentamicin, cefoxitin, ceftazidime, } \\
\text { piperacillin. Sensitive to cefepime, } \\
\text { levofloxacin }\end{array}$ & ND & ND & $\begin{array}{l}\text { Guo and } \\
\text { Liu, } \\
2011\end{array}$ \\
\hline 5 & $30 / \mathrm{F}$ & Burn & $\begin{array}{l}\text { Blood, central } \\
\text { venous catheter, } \\
\text { urine }\end{array}$ & Sensitive to amikcin, norfloxacin & Amikacin & Favorable & Wu, 1998 \\
\hline 6 & $28 / \mathrm{F}$ & $\begin{array}{l}\text { Injury and } \\
\text { surgery }\end{array}$ & Wound & $\begin{array}{l}\text { Resistant to gentamicin, sulfamethoxazole, } \\
\text { ciprofloxacin, cefoperazone-sulbactam, } \\
\text { tetracycline, tobramycin, cefoperazone, } \\
\text { cefepime, imipenem, piperacillin-tazobactam, } \\
\text { cefoselis, amikacin, piperacillin, } \\
\text { levofloxacin, netilmicin, ceftazidime, } \\
\text { cefotaxime, aztreonam, ampicillin-sulbactam. } \\
\text { Sensitive to minocycline. Moderately } \\
\text { sensitive to meropenem }\end{array}$ & $\begin{array}{l}\text { Debridement, skin } \\
\text { transplantation, iv } \\
\text { cefperazone- } \\
\text { sulbactam and } \\
\text { oral minocycline } \\
\text { for } 3 \mathrm{~d} \text {, then oral } \\
\text { minocycline for } \\
\text { another } 3 \mathrm{~d}\end{array}$ & Cured & $\begin{array}{l}\text { Hu et al., } \\
2013\end{array}$ \\
\hline 7 & $76 / \mathrm{M}$ & $\begin{array}{l}\text { Chronic } \\
\text { obstructive } \\
\text { pulmonary } \\
\text { disease and } \\
\text { heart failure, } \\
\text { old age }\end{array}$ & Blood, wound & $\begin{array}{l}\text { Resistant to piperacillin, ceftazidime, } \\
\text { ceftriaxone, cefepime, aztreonam, } \\
\text { imipenem, meropenem, amikacin, } \\
\text { gentamicin, ciprofloxacin, levofloxacin, } \\
\text { tetracycline, trimethoprim-sulfamethoxazole, } \\
\text { ampicillin-sulbactam, cefoperazone- } \\
\text { sulbactam, piperacillin-tazobactam }\end{array}$ & $\begin{array}{l}\text { Oral minocycline } \\
\quad \text { for } 9 \mathrm{~d}\end{array}$ & Cured & $\begin{array}{l}\text { Huang } \\
\text { et al., } \\
2014\end{array}$ \\
\hline 8 & $4 / \mathrm{M}$ & None & Blood & $\begin{array}{l}\text { Resistant to ampicillin, ampicillin- } \\
\text { sulbactam, piperacillin, piperacillin } \\
\text { tazobactam, aztreonam, cefazolin, } \\
\text { cefoxitin, ceftazidime, cefotaxime, azole } \\
\text { cefepime, ceftazidime, ceftriaxone, } \\
\text { cefepime }\end{array}$ & $\begin{array}{l}\text { Piperacillin and } \\
\text { tobramycin for } \\
14 \mathrm{~d}\end{array}$ & Cured & $\begin{array}{l}\text { Huang } \\
\text { and Lin, } \\
2003\end{array}$ \\
\hline 9 & $58 / \mathrm{ND}$ & $\begin{array}{l}\text { Diabetes } \\
\text { mellitus } \\
\text { complicated } \\
\text { by heel } \\
\text { bursitis }\end{array}$ & Drainage & Sensitive to cefoperazone and amikacin & $\begin{array}{l}\text { Incision and drainage, } \\
\text { cefoperazone and } \\
\text { amikacin for } \\
\text { several days } \\
\text { (more than } 3 \mathrm{~d} \text { ) }\end{array}$ & Cured & $\begin{array}{l}\text { Yang and } \\
\text { Wang, } \\
2001\end{array}$ \\
\hline 10 & $28 / \mathrm{F}$ & None & Pus & $\begin{array}{l}\text { Resistant to kanamycin, penicillin, } \\
\text { erythromycin. Sensitive to ceftriaxone, } \\
\text { norfloxacin, trimethoprim-sulfamethoxazole }\end{array}$ & $\begin{array}{l}\text { Abscess incision } \\
\text { drainage and } \\
\text { norfloxacin and } \\
\text { trimethoprim- } \\
\text { sulfamethoxazole }\end{array}$ & Cured & $\begin{array}{l}\text { Song } \\
\text { et al., } \\
1995\end{array}$ \\
\hline
\end{tabular}


Table 1

\begin{tabular}{|c|c|c|c|c|c|c|c|}
\hline $\begin{array}{l}\text { Patient } \\
\text { No. }\end{array}$ & $\begin{array}{c}\text { Age } \\
\text { (year)/ } \\
\text { gender }\end{array}$ & $\begin{array}{l}\text { Underlying } \\
\text { diseases or } \\
\text { reasons }\end{array}$ & Site of isolation & Antibiotic resistance status & Treatment strategy & Outcome & Reference \\
\hline 11 & $24 \mathrm{~d} / \mathrm{M}$ & Neonate & Blood & $\begin{array}{l}\text { Resistant to penicillin, chloramphenicol, } \\
\text { carbenicillin, streptomycin, cefazolin. } \\
\text { Sensitive to amikacin, erythromycin, } \\
\text { ampicillin, benzylpencilline }\end{array}$ & $\begin{array}{l}\text { Ampicillin, and } \\
\text { oxacillin for } 19 \mathrm{~d}\end{array}$ & Cured & $\begin{array}{l}\text { Wang and } \\
\quad \text { Su, } \\
1992\end{array}$ \\
\hline 12 & $11 \mathrm{~d} / \mathrm{M}$ & Preterm birth & Blood, CSF & $\begin{array}{l}\text { Resistant to ampicillin, cefazolin, } \\
\text { gentamicin, cefoperazone, cefotaxime, } \\
\text { cefatrizine, ceftazidime. Sensitive to } \\
\text { amikacin, piperacillin, ampicillin, } \\
\text { sulbactam-cefoperazone }\end{array}$ & $\begin{array}{l}\text { Antimicrobial } \\
\text { treatment for } 5 \mathrm{~d} \\
\text { (the antibiotic was } \\
\text { not described) }\end{array}$ & Failed & $\begin{array}{l}\text { Zhang and } \\
\text { Zhang, } \\
1996\end{array}$ \\
\hline 13 & $69 / \mathrm{F}$ & $\begin{array}{l}\text { Lung cancer } \\
\text { and surgery, } \\
\text { old age }\end{array}$ & $\begin{array}{l}\text { Pleural effusion } \\
\text { and sputum }\end{array}$ & $\begin{array}{l}\text { Resistant to tobramycin, gentamicin, } \\
\text { ampicillin, erythromycin, clindamycin, } \\
\text { tetracycline. Sensitive to amikacin, } \\
\text { tobramycin, ceftriaxone }\end{array}$ & $\begin{array}{l}\text { Antimicrobial } \\
\text { treatment, but not } \\
\text { described in detail }\end{array}$ & Died & $\begin{array}{l}\text { Song, } \\
2005\end{array}$ \\
\hline 141 & $\begin{array}{l}10 \text { months } / \\
\mathrm{F}\end{array}$ & / Child & Blood & $\begin{array}{l}\text { Sensitive gentamicin, tobramycin, } \\
\text { cephalexin, sulbactam-cefoperazone, } \\
\text { ceftriaxone }\end{array}$ & $\begin{array}{l}\text { Cefoperazone, } \\
\text { tobramycin for } \\
10 \mathrm{~d}\end{array}$ & Cured & $\begin{array}{l}\text { Zhao, } \\
2000\end{array}$ \\
\hline 15 & $60 / \mathrm{M}$ & $\begin{array}{r}\text { Common bile } \\
\text { duct stones }\end{array}$ & $\begin{array}{l}\text { Blood, bile, } \\
\text { peritoneal } \\
\text { effusion }\end{array}$ & $\begin{array}{l}\text { Resistant to tobramycin. Sensitive to } \\
\text { piperacillin, cefoperazone, amikacin, } \\
\text { gentamicin, ceftriaxone, cefotaxime }\end{array}$ & $\begin{array}{l}\text { Amikacin and } \\
\text { cefoperazone }\end{array}$ & Cured & $\begin{array}{l}\text { Meng } \\
\text { et al., } \\
1999\end{array}$ \\
\hline 16 & $44 / F$ & None & $\begin{array}{l}\text { Blood, bone } \\
\text { marrow }\end{array}$ & $\begin{array}{l}\text { Sensitive to norfloxacin, ciprofloxacin, } \\
\text { cefazolin, amikacin, ceftazidime }\end{array}$ & ND & ND & $\begin{array}{l}\text { Geng et al., } \\
\quad 2000\end{array}$ \\
\hline 17 & $67 / \mathrm{M}$ & Old age & $\begin{array}{l}\text { Sputum (this strain } \\
\text { was isolated } \\
\text { with Serratia } \\
\text { marcescens, } \\
\text { Acinetobacter } \\
\text { lwoffi) }\end{array}$ & $\begin{array}{l}\text { Resistant to ampicillin, piperacillin } \\
\text { cefazolin, cefuroxime, cefotaxime, } \\
\text { ceftazidime, cefotaxime, aztreonam, } \\
\text { gentamicin, norfloxacin, } \\
\text { trimethoprim-sulfamethoxazole }\end{array}$ & $\begin{array}{l}\text { Antimicrobial } \\
\text { treatment, but not } \\
\text { described in detail }\end{array}$ & Cured & $\begin{array}{l}\text { Liu and } \\
\text { He, } \\
2001\end{array}$ \\
\hline 18 & $45 / M$ & None & Urine & $\begin{array}{l}\text { Resistant to ampicillin, amikacin, } \\
\text { azithromycin }\end{array}$ & $\begin{array}{l}\text { Application of } \\
\text { cefoperazone, } \\
\text { cefotaxime, } \\
\text { nitrofurantoin, } \\
\text { and tobramycin } \\
\text { for } 3 \text { weeks }\end{array}$ & Cured & $\begin{array}{l}\text { Wuer } \\
\text { et al., } \\
2000\end{array}$ \\
\hline 19 & N/A & ND & $\begin{array}{l}\text { Blood, sputum, } \\
\text { bile, cerebrospinal } \\
\text { fluid, urine, all } \\
\text { these three } \\
\text { isolates were } \\
\text { from patients (no } \\
\text { further details } \\
\text { were available) }\end{array}$ & $\begin{array}{l}\text { All } 12 \text { isolates were resistant to } \\
\text { erythromycin, penicillin, streptomycin, } \\
\text { ampicillin, oxacillin, piperacillin, } \\
\text { carbenicillin }\end{array}$ & N/A & N/A & $\begin{array}{l}\text { Li and } \\
\text { Zhao, } \\
1995\end{array}$ \\
\hline 20 & ND & $\begin{array}{l}\text { Chronic } \\
\text { nephritis }\end{array}$ & Urine & $\begin{array}{l}\text { Two isolates were resistant to meropenem. } \\
\text { All three isolates were resistant to }\end{array}$ & $\begin{array}{l}\text { One isolate was } \\
\text { sensitive to }\end{array}$ & ND & $\begin{array}{l}\text { Li et al., } \\
\quad 2010\end{array}$ \\
\hline 21 & ND & $\begin{array}{l}\text { Diabetes } \\
\text { mellitus }\end{array}$ & & & meropenem & & \\
\hline 22 & $\mathrm{ND} / \mathrm{F}$ & $\begin{array}{l}\text { Cervical } \\
\text { cancer }\end{array}$ & & $\begin{array}{l}\text { ceftriaxone, aztreonam, gentamicin, } \\
\text { ciprofloxacin, levofloxacin, ampicillin, } \\
\text { piperacillin, cefazolin, cefuroxime axetil, } \\
\text { ceftazidime, cefepime, imipenem, } \\
\text { amikacin, tobramycin, levofloxacin, } \\
\text { trimethoprim-sulfamethoxazole }\end{array}$ & & & \\
\hline 23 & $61 / \mathrm{F}$ & $\begin{array}{l}\text { Coma, } \\
\quad \text { cerebral } \\
\text { hemorrhage }\end{array}$ & Sputum & N/A & $\begin{array}{l}\text { Ceftazidine, } \\
\text { chloramphenicol, } \\
\text { penicillin } \mathrm{G}, \\
\text { gentamicin by } \\
\text { atomization } \\
\text { inhalation, } \\
\text { ketoconazole by } \\
\text { nasal feeding }\end{array}$ & Died & $\begin{array}{l}\text { Jin and } \\
\text { Xiao, } \\
1995\end{array}$ \\
\hline
\end{tabular}


Table 1

\begin{tabular}{|c|c|c|c|c|c|c|c|}
\hline $\begin{array}{l}\text { Patient } \\
\text { No. }\end{array}$ & $\begin{array}{c}\text { Age } \\
\text { (year)/ } \\
\text { gender }\end{array}$ & $\begin{array}{l}\text { Underlying } \\
\text { diseases or } \\
\text { reasons }\end{array}$ & Site of isolation & Antibiotic resistance status & Treatment strategy & Outcome & Reference \\
\hline 24 & $49 / \mathrm{M}$ & $\begin{array}{l}\text { Chronic alcohol } \\
\text { misuse }\end{array}$ & Blood & Intermediately sensitive to imipenem & $\begin{array}{l}\text { Treatment with } \\
\text { amoxicillin- } \\
\text { clavulanic acid was } \\
\text { changed to } \\
\text { ciprofloxacin, } \\
\text { imipenem-cilastatin } \\
\text { used for } 10 \mathrm{~d} \text {, then } \\
\text { oral ciprofloxacin } \\
\text { for } 21 \mathrm{~d}\end{array}$ & Cured & $\begin{array}{l}\text { Bachmeyer } \\
\text { et al., } \\
2007\end{array}$ \\
\hline 25 & $55 / \mathrm{F}$ & $\begin{array}{l}\text { Liver cirrhosis } \\
\text { bilateral lower } \\
\text { extremity } \\
\text { cellulitis and } \\
\text { open wounds }\end{array}$ & Blood, wound & $\begin{array}{l}\text { Resistant to amikacin, gentamicin, } \\
\text { tobramycin, aztreonam, ceftriaxone, } \\
\text { ciprofloxacin, tetracycline, } \\
\text { trimethoprim-sulfamethoxazole, } \\
\text { vancomycin. Intermediately sensitive } \\
\text { to piperacillin-tazobactam, cefepime, } \\
\text { imipenem, and cilastatin }\end{array}$ & $\begin{array}{l}\text { iv vancomycin, } \\
\text { piperacillin- } \\
\text { tazobactam, and } \\
\text { levofloxacin for } \\
18 \mathrm{~h} \text {, then iv } \\
\text { imipenem-cilastatin, } \\
\text { daptomycin, } \\
\text { clindamycin, then } \\
\text { imipenem-cilastatin } \\
\text { and doxycycline }\end{array}$ & Died & $\begin{array}{l}\text { Crum- } \\
\text { Cianflone } \\
\text { et al., } \\
2014\end{array}$ \\
\hline 26 & $13 / \mathrm{M}$ & $\begin{array}{l}\text { Soft tissue } \\
\text { infection }\end{array}$ & Pus & $\begin{array}{l}\text { Resistant to piperacillin-tazobactam, } \\
\text { aztreonamaminoglycosides. } \\
\text { Intermediately susceptible to } \\
\text { imipenem. Sensitive to all quinolones } \\
\text { tested, cotrimoxazole, } \\
\text { chloramphenicol, and } \\
\text { amoxicillin-clavulanic acid }\end{array}$ & $\begin{array}{l}\text { Drainage of osteolytic } \\
\text { lesions combined } \\
\text { with iv ciprofloxacin } \\
\text { for } 10 \mathrm{~d} \text { and } \\
\text { continued with oral } \\
\text { ciprofloxacin for an } \\
\text { additional } 10 \mathrm{~d}\end{array}$ & Cured & $\begin{array}{c}\text { Maraki } \\
\text { et al., } \\
2012\end{array}$ \\
\hline 27 & $48 / \mathrm{F}$ & $\begin{array}{l}\text { Cystitis } \\
\quad(\text { contaminated })\end{array}$ & Urine & $\begin{array}{l}\text { Fully resistant to streptomycin, } \\
\text { gentamicin, kanamycin, ampicillin, }\end{array}$ & N/A & N/A & $\begin{array}{l}\text { Holmes } \\
\text { et al., }\end{array}$ \\
\hline 28 & $34 / \mathrm{M}$ & $\begin{array}{l}\text { Infected cut } \\
\text { finger }\end{array}$ & Wound & $\begin{array}{l}\text { carbenicillin, tetracycline, } \\
\text { polymyxin } \mathrm{B} \text {. }\end{array}$ & & & 1977 \\
\hline 29 & $59 / \mathrm{F}$ & ND & Urine & Fully resistant or moderately resistant to & & & \\
\hline 30 & & $\begin{array}{l}\text { Urinary } \\
\text { retention }\end{array}$ & Urine & $\begin{array}{l}\text { sulfamethoxazole, trimethoprim- } \\
\text { sulfamethoxazole, cephaloridine, }\end{array}$ & & & \\
\hline 31 & & $\begin{array}{l}\text { Further details } \\
\text { are not } \\
\text { available }\end{array}$ & Urine & $\begin{array}{l}\text { erythromycin, chloramphenicol. } \\
\text { Moderately sensitive to nalidixic acid }\end{array}$ & & & \\
\hline 32 & $\mathrm{ND} / \mathrm{ND}$ & Varicose ulcer & Wound & & & & \\
\hline 33 & $76 / \mathrm{F}$ & Leg ulcer & Ulcer & & & & \\
\hline 34 & $67 / \mathrm{F}$ & Breast lump & Urine & & & & \\
\hline 35 & 48/M & $\begin{array}{l}\text { Chronic renal } \\
\text { insufficiency }\end{array}$ & Urine & & & & \\
\hline 36 & $66 / \mathrm{M}$ & $\begin{array}{l}\text { Urinary tract } \\
\text { infection }\end{array}$ & Urine & $\begin{array}{l}\text { Resistant to all } \beta \text {-lactam and non- } \\
\quad \beta \text {-lactam antibiotics tested, including }\end{array}$ & Imipenem, colistin & Failure & $\begin{array}{l}\text { Ktari et al., } \\
\quad 2012\end{array}$ \\
\hline 37 & $44 / \mathrm{M}$ & Bladder & Urine & imipenem, vancomycin, & No treatment & Favorable & \\
\hline 38 & $44 / \mathrm{M}$ & colonization & Urine & ciprofloxacin, chloramphenicol, & No treatment & Favorable & \\
\hline 39 & $47 / \mathrm{M}$ & & Urine & tigecycline, rifampicin & No treatment & Favorable & \\
\hline 40 & 77/M & $\begin{array}{l}\text { Urinary tract } \\
\text { infection }\end{array}$ & Urine & & $\begin{array}{l}\text { Ifampicinp } \\
\text { ciprofloxacin }\end{array}$ & Cured & \\
\hline 41 & $65 / \mathrm{M}$ & & Urine & & $\begin{array}{l}\text { Ifampicinp } \\
\text { ciprofloxacin }\end{array}$ & Cured & \\
\hline 42 & $80 / \mathrm{M}$ & & Urine & & $\begin{array}{l}\text { Ifampicinp } \\
\text { ciprofloxacin }\end{array}$ & Cured & \\
\hline 43 & $59 / \mathrm{M}$ & $\begin{array}{l}\text { Urinary tract } \\
\text { infection }\end{array}$ & Urine & $\begin{array}{l}\text { Resistant to amikacin, gentamicin, } \\
\text { imipenem, meropenem, cefazolin, } \\
\text { ceftazidime, cefotaxime, cefepime, } \\
\text { aztreonam, ampicilllin, piperacillin, } \\
\text { amoxicillin-clavulanate, ampicillin- } \\
\text { sulbactam, piperacillin-tazobactam, } \\
\text { colistin, trimethoprim- } \\
\text { sulfamethoxazole, chloramphenicol, } \\
\text { ciprofloxacin, levofloxacin, } \\
\text { moxifloxacin, tetracycline }\end{array}$ & $\begin{array}{l}\text { Levofloxacin was used } \\
\text { only temporarily } \\
\text { and orally }\end{array}$ & Failure & Our case \\
\hline
\end{tabular}

M: male; F: female; N/A: not applicable; ND: not described; CSF: cerebrospinal fluid; iv: intravenous injection 
Table 2 Antimicrobial susceptibility testing of 23 strains of Myroides sp. using the K-B method

\begin{tabular}{llccc}
\hline Site of isolation (total samples of positive isolation) & Antibiotic & $R$ & $I$ & $S$ \\
\hline Sputum (8); & Amikacin & 5 & 10 & 8 \\
Urine (6); & Cefazolin & 11 & 9 & 3 \\
Blood (4); & Cefoperazone & 9 & 9 & 5 \\
CSF (3); & Sulfamethoxazole & 4 & 10 & 9 \\
Bile (2) & Sulfadiazine & 5 & 7 & 11 \\
& Ceftazidime & 10 & 6 & 7 \\
& Erythrocin & 3 & 10 & 10 \\
& Azithromycin & 4 & 9 & 10 \\
\hline
\end{tabular}

Translated from Lan and Bao (2009) with permission of the authors. K-B method: Kirby-Bauer disk diffusion method; CSF: cerebrospinal fluid; $R$ : resistant; $I$ : immediately sensitive; $S$ : sensitive

Table 3 Antimicrobial susceptibility testing of 11 strains of Myroides sp. isolated from urine using Oxoid culture medium

\begin{tabular}{|c|c|c|c|c|}
\hline Patient information & Antibiotic & $R$ & $I$ & $S$ \\
\hline \multirow{4}{*}{$\begin{array}{l}\text { 2-76 years (average } 53 \text { years), } 9 \text { males, } 2 \text { females } \\
\text { All patients suffered from urinary retention or } \\
\text { urinary tract stones, but none of them had } \\
\text { symptoms of urinary tract infection or other } \\
\text { discomfort }\end{array}$} & Ampicillin & 11 & 0 & 0 \\
\hline & Piperacillin & 11 & 0 & 0 \\
\hline & Cefuroxime & 11 & 0 & 0 \\
\hline & Cefoperazone-sulbactam & 11 & 0 & 0 \\
\hline \multirow{4}{*}{$\begin{array}{l}\text { In nine urinarily catheterized patients, the urinary } \\
\text { culture when the catheter was in situ was Myroides } \\
\text { sp. positive, but the urinary testing showed no } \\
\text { WBC in these urinary samples, and pus cells were } \\
\text { found in only three of them }\end{array}$} & Ceftazidine & 10 & 1 & 0 \\
\hline & Cefepime & 10 & 1 & 0 \\
\hline & Aztreonam & 11 & 0 & 0 \\
\hline & Imipenem & 11 & 0 & 0 \\
\hline \multirow{5}{*}{$\begin{array}{l}\text { The urinary culture of Myroides } \mathrm{sp} \text {. became negative } \\
\text { after removal of urinary catheter in these nine } \\
\text { urinarily catheterized patients even though they } \\
\text { were not treated }\end{array}$} & Meropenem & 11 & 0 & 0 \\
\hline & Levofloxacin & 8 & 3 & 0 \\
\hline & Ciprofloxacin & 9 & 2 & 0 \\
\hline & Trimethoprim-sulphamethoxazole & 0 & 0 & 11 \\
\hline & Amikacin & 11 & 0 & 0 \\
\hline
\end{tabular}

Translated from Chen et al. (2009) with permission of Chin. J. Pract. Med. Tech. R: resistant; I: immediately sensitive; S: sensitive; WBC: white blood cell

\section{Preliminary opinions on the antibiotic re- sistance mechanisms of Myroides sp.}

In China, there have been no reports on the antibiotic resistance mechanisms of Myroides sp. Although several foreign researchers have investigated this topic, very little information is available. Hummel et al. (2007) showed that the $\beta$-lactamase gene was responsible for the variable patterns of resistance to $\beta$-lactam antibiotics and the decreased susceptibility to carbapenems of different Myroides sp. strains. In a study investigating a number of clinical cases involving systemic infections, Mammeri et al. (2002) claimed that resistance to $\beta$-lactams was due to the production of the chromosome-encoded $\beta$-lactamases TUS- 1 and MUS-1 in M. odoratus and M. odoratimimus. The $\beta$-lactamases produced by Gram-negative and Gram-positive bacteria play a vital role in resistance against $\beta$-lactam antibiotics. However, their study showed that the $\beta$-lactamases TUS- 1 and MUS-1 could only partly explain the intrinsic resistance of Flavobacteriaceae species to $\beta$-lactams. Also, a common observation was that these Escherichia coli expressed metalloenzymes were much 
less resistant to $\beta$-lactam than those of primitive origin (Mammeri et al., 2002). Even Flavobacteriaceae and Myroides sp. belong to the same family, the mechanism of resistance conferred by TUS- 1 and MUS-1 in Flavobacteriaceae species cannot be assumed to operate in Myroides sp. Then, why does Flavobacteriaceae serve as a source for a variety of metalloenzymes? As observed for other environmental species, this might be due to the combined biosynthesis of carbapenem derivatives and hydrolyzing $\beta$-lactamases (Mammeri et al., 2002).

Suganthi et al. (2013) investigated whether the antibiotic sensitivity of plasmid-containing $M$. odoratimimus SKS05-GRD was correlated with the plasmid or was chromosomally-mediated. They revealed that resistance to kanamycin, amikacin, and gentamicin was plasmid-mediated, and that resistance to ampicillin, cefadroxil, cefoperazone, ceftazidine, ceftriaxone, and netillin was chromosomally-mediated. The Klebsiella pneumoniae carbapenemase (KPC) family is closely related to resistance to carbapenem in a variety of pathogens and the KPC gene is located in a plasmid. However, Myroides sp. WX2856, obtained from an abdominal abscess (Kuai et al., 2011), harbored a KPC-2 carbapenemase, but the $K P C-2$ gene might not be located on a plasmid as in $K$. pneumoniae. Do these results suggest the possibility of interspecies transmission of the KPC-2 gene? This aspect needs further investigation.

On the other hand, there are several known features of antibiotic resistance mechanisms in bacteria from the same family of Myroides, such as in F. indologenes, now named Chryserobacterium indologenes (Tian and Wang, 2010). First, resistance transfer factors (R-factors) in the cytoplasm determine the bacteria's resistance to antibiotics. R-factor plasmids can carry and transfer a variety of resistance genes among bacteria. In addition, the thick outer membrane and its low permeability, resulting from multidirectional mutations, and the active discharge system of the bacterial cell membrane of $C$. indologenes confer inherent multi-drug resistance. The bacteria also produce a $\beta$-lactamase with a broad spectrum of $\beta$-lactam hydrolytic activity (Tian and Wang, 2010).

Thus, it is apparent that the antibiotic resistance mechanisms of Myroides sp. are unclear and deserve further investigation.

\section{Present diagnostic methods do not clarify the antibiotic resistance mechanisms of Myroides sp.}

In clinical diagnostic laboratories, traditional bacterial identification methods primarily rely on testing biological traits and biochemical characteristics, and include microscopic inspection and metabolic testing of isolated and cultured bacteria. These methods have shown that Myroides spp. do not have flagella, release a fruity fragrance, are yellow, oxidasepositive, urea- and indole-negative, and are unable to oxidize sugar (Li and Zhao, 1995; Chen et al., 2009). However, the bacterial strain can be preliminarily identified only as Myroides sp., as further strain type designations cannot be determined using these traditional methods. Nearly all cases of Myroides sp. infections reported in China have used these traditional identification approaches, and did not describe any Myroides sp. subtypes. Using these traditional identification methods, the $M$. odoratimimus strain PR63039 isolated in our case was first identified as Pseudomonas putida, then as M. odoratimimus, and as the Acinetobacter calcoaceticus-baumannii complex, at different time points throughout the $47 \mathrm{~d}$ of the patient's hospitalization. It was finally confirmed as $M$. odoratimimus by $16 \mathrm{~S}$ rRNA sequencing. This case also indicates that these traditional identification methods may not correctly diagnose the strain type.

Recently, other microorganism strain identification technologies have been developed, including VITEK 2 (bioMerieux VITEK-2, France), matrixassisted laser desorption/ionization time-of-flight mass spectrometry (MALDI-TOF MS), 16S rDNA sequencing, and another more frequently used nomenclature, 16S rRNA sequencing (Table 4). VITEK 2, a routine laboratory method, can help to discriminate among genera but not among species. MALDI-TOF MS and 16S rDNA sequencing/16S rRNA sequencing can be used to identify species and are more frequently used for research purposes (Lee et al., 2014; Schröttner et al., 2014). According to Schröttner et al. (2014), the genus Myroides was reliably identified in tests of 22 isolates using VITEK 2. 16S rDNA sequencing further revealed that they shared $\geq 97 \%$ homology, enough for a reliable identification at the species level. Yoon et al. (2006) applied 16S rRNA sequencing successfully to clarify the phylogenetic 
position of Myroides sp. strain SM1T, isolated from seawater in Thailand.

Table 4 Comparison of the present approaches for strain identification of Myroides sp."

\begin{tabular}{cc}
\hline \multicolumn{1}{c}{ Method } & Trait \\
\hline VITEK2 & $\begin{array}{c}\text { Only suitable to identify bacteria at } \\
\text { the genus level, not at the species } \\
\text { level }\end{array}$ \\
MALDI-TOF MS & $\begin{array}{c}\text { Able to distinguish between } M . \\
\text { odoratus } \text { and } \text { M. odoratimimus }\end{array}$ \\
16S rDNA & $\begin{array}{c}\text { Able to distinguish microorganisms } \\
\text { sequencing/16S } \\
\text { rRNA sequencing }\end{array}$ \\
$\begin{array}{c}\text { Whole genome } \\
\text { sequencing }\end{array}$ & $\begin{array}{c}\text { Able to identify the microorganism } \\
\text { and provide the bioinformatics of } \\
\text { microorganism }\end{array}$ \\
\hline
\end{tabular}

*Yoon et al., 2006; Lee et al., 2014; Schröttner et al., 2014

\section{Whole genome sequencing is a feasible way to investigate the antibiotic resistance mechanisms of Myroides sp.}

In the past decade, many next generation sequencing platforms have been developed, such as 454 invented in 2004 (Margulies et al., 2005), Illumina Solexa in 2006 (Bentley, 2006), SOLiD in 2007 (Chi, 2008), Ion Torrent in 2011 (Rothberg et al., 2011), and PacBio in 2012 (Koren et al., 2012). This has led to a rapid increase in the sequencing of whole genomes of microorganisms, including eukarya, bacteria, archaea, and viruses. These sequences have been deposited in the National Center for Biotechnology Information (NCBI) RefSeq genome collection database (http://www.ncbi.nlm.nih.gov/genome) (Tatusova et al., 2015). In 2014, over 10000 microbial genomes were released (Tatusova et al., 2015). Along with the next generation platforms, wholegenome analysis of multi-drug resistance mechanisms has emerged.

For example, A. baumannii is a common cause of fatal nosocomial infections because of its extensive antibiotic resistance. Genomic sequencing revealed comprehensive drug-resistance mechanisms, such as a 41.6-kb closely related antibiotic resistance island in the chromosome (Huang et al., 2012), the horizontally transmittable carbapenem resistance gene $\left(b l a_{\text {OXA-23 }}\right)$ containing a plasmid (in isolate MDR-TJ, with 454 Titanium) among different $A$. baumannii strains (Huang et al., 2012; Lee et al., 2013), a list of antimicrobial resistance-associated genes (with 454 and SOLiD) (Rolain et al., 2013), a diversified resistance gene list (with Illumina Hiseq2000) (Tan et al., 2013), and longitudinally evolved antibiotic resistance gene mutations and mutational pathways under pressure from the antibiotic colistin (with 454 Titanium) (Snitkin et al., 2013). Since its invention by Rothberg et al. (2011), Ion Torrent sequencing technology has been successfully applied to complete genomic sequencing, such as in Clostridium sp. BL8 (with Ion Torrent PGM ${ }^{\mathrm{TM}}$ ) (Marathe et al., 2014), and clear characterization of drug-resistant genes, such as in Mycobacterium tuberculosis (Daum et al., 2014). Results can be obtained within five days, comparable to the turnaround time required by current drug sensitivity testing (DST) (Daum et al., 2014). PacBio single-molecule real-time technology has frequently been used to perform whole-genome sequencing of many microorganisms, such as Neisseria gonorrhea (with the PacBio RSII platform), a Gram-negative $\beta$ proteobacterium responsible for the sexually transmitted infection gonorrhea (Abrams et al., 2015).

Yet, little genomic information about Myroides $\mathrm{sp}$. is available. A brief description of the genomes of M. odoratus DSM 2801 and CIP 103059 was found in genome database of NCBI (Table 5), but this was not suitable for studying its drug-resistance mechanisms. Recently, the genome sequencing of the urethral catheter isolate Myroides sp. A21 was completed (Burghartz et al., 2015). The sequence contained 3650 protein-coding sequences (CDSs), 136 RNAcoding genes, and eight copies of the rRNA gene cluster, of which three were resolved as a direct repeat of two rRNA gene clusters. The presence of 106 transfer RNAs (tRNAs) and six noncoding RNAs (ncRNAs) was also predicted. By comparing the genome sequence of Myroides sp. A21 with those of $M$. odoratimimus CCUG 10230 and M. odoratus DSM 2801, 293 unique CDSs were found in the A21 genome (Burghartz et al., 2015). In addition, five genomic islands were predicted by Island Viewer analysis (Burghartz et al., 2015). However, as the antibiotic treatment history was not described and the antibiotic resistance status of this strain was not given, the antibiotic resistance mechanisms could not be analyzed from the data. 
Table 5 Reported RefSeq genome of Myroides odoratus CIP 103059 and DSM 2801

\begin{tabular}{cccccccccccc}
\hline Strain & Name & RefSeq & INSDC & $\begin{array}{c}\text { Size } \\
(\mathrm{Mb})\end{array}$ & $\begin{array}{c}\text { Total } \\
\text { number of } \\
\text { genes }\end{array}$ & $\begin{array}{c}\text { Total } \\
\text { number of } \\
\text { proteins }\end{array}$ & rRNA & tRNA & $\begin{array}{c}\text { Other } \\
\text { genes }\end{array}$ & $\begin{array}{c}\text { GC } \\
\text { content } \\
(\%)\end{array}$ & Pseudogenes \\
\hline CIP 103059 & $\begin{array}{c}\text { Master } \\
\text { WGS NZ_AGZJ000000 AGZJ000000 }\end{array}$ & $\begin{array}{c}4.23 \\
00.1\end{array}$ & 3773 & 3631 & 10 & 67 & 1 & 35.8 & 64 \\
DSM 2801 & & NZ_CM001437.1 & $\begin{array}{c}00.1 \\
\text { CM001437.1 }\end{array}$ & 4.3 & 3838 & 3695 & 9 & 74 & 1 & 35.8 & 59 \\
\hline
\end{tabular}

Cited from GenBank assembly accession: GCA_000243275.1 and GCA_000297875.1. INSDC: International Nucleotide Sequence Database Collaboration

To study these aspects in our M. odoratimimus strain PR63039, we extracted its genomic DNA. Agarose gel electrophoresis results from several experiments revealed that it might harbor at least six different types of plasmids which could be related to antibiotic resistance (Fig. 1). However, the exact number of plasmid type needs further confirmation. Many bacterial drug-resistance genes are plasmid- or chromosome-mediated, but we did not know which mechanism was operating in Myroides sp. The genome of strain PR63039 was sequenced using an Ion Torrent Personal Genome Machine. We generated 610 contigs and 4221 open reading frames. The total sequence numbers with Gene Ontology (GO) and Clusters of Orthologous Groups (COG) of protein were 2741 and 1026, respectively. However, we could not completely assemble the genome and plasmids, so the multi-drug resistance mechanisms of strain PR63039 still could not be clarified. We are now using PacBio single-molecule real-time technology in the hope of generating a complete genome sequence of both the chromosome and plasmids, to elucidate the mechanisms of resistance and pathogenesis of this strain.

All the infection reports from China indicated that Myroides sp. is a serious source of nosocomial infection and has the potential to cause a pandemic. The completion of the Myroides sp. genome sequence and detailed bioinformatics analysis are imperative for the understanding of its mechanisms of antibiotic resistance and pathogenesis.

\section{Discussion and outlook}

Myroides sp. is an opportunistic and extensively antibiotic-resistant pathogen. Infections have not been widely reported, though there have been many cases in China. As the antibiotic resistance mechanisms of Myroides sp. are still unclear, and in view of the risk of nosocomial infection and pandemics,

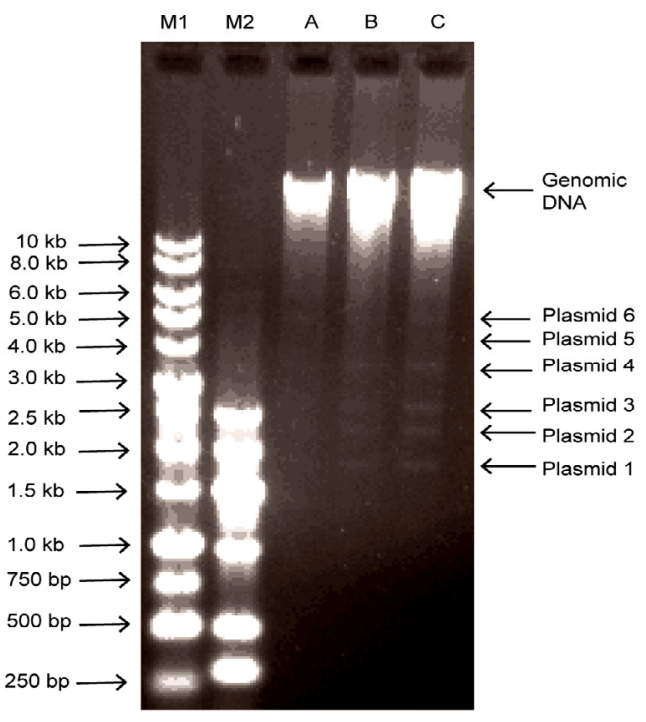

Fig. 1 Agarose gel electrophoresis of genomic DNA of Myroides odoratimimus strain PR63039

The genomic DNA of PR63039 was separated by gel electrophoresis using $0.75 \%(7.5 \mathrm{~g} / \mathrm{ml})$ agarose. The genomic DNA was about $23 \mathrm{~kb}$ in size. M1: $1 \mathrm{~kb}$ DNA marker; M2: DNA marker-G; A: $0.173 \mu \mathrm{g}$ DNA; B: $0.273 \mu \mathrm{g}$ DNA; C: $0.328 \mu \mathrm{g}$ DNA. The genomic DNA was about $23 \mathrm{~kb}$ in size. Six different types of plasmids (1-6) were visible

novel technologies, such as whole genome sequencing and further bioinformatic analyses, should be applied urgently to Myroides sp. An outline of a strategy for whole genome sequencing and bioinformatic analyses is presented in Fig. 2. These analyses will also be helpful in developing appropriate management strategies. Moreover, whole genome sequencing might become a routine diagnosis method for all microbial infections in the near future.

\section{Compliance with ethics guidelines}

Shao-hua HU, Shu-xing YUAN, Hai QU, Tao JIANG, Ya-jun ZHOU, Ming-xi WANG, and De-song MING declare that they have no conflict of interest.

This article does not contain any studies with human or animal subjects performed by any of the authors. 


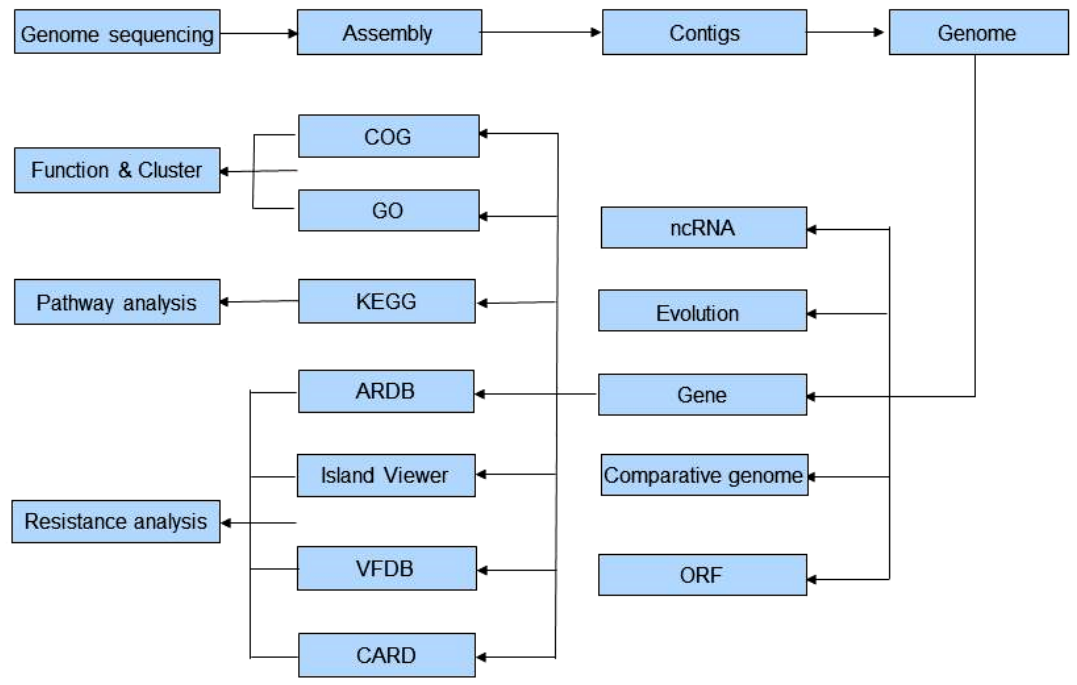

Fig. 2 Procedure of the strategy of whole genome sequencing and bioinformatics analyses

COG: Clusters of Orthologous Group; GO: Gene Ontology; KEGG: kyoto encyclopedia of genes and genome; ARDB: Antibiotic Resistance Genes Database; VFDB: Virulence Factor Database; CARD: Comprehensive Antibiotic Research Database; ncRNA: noncoding RNA; ORF: open reading frame

\section{References}

Abrams, A.J., Trees, D.L., Nicholas, R.A., 2015. Complete genome sequences of three Neisseria gonorrhoeae laboratory reference strains, determined using PacBio single-molecule real-time technology. Genome Announc., 3(5):e01052-15.

http://dx.doi.org/10.1128/genomeA.01052-15

An, R.F., 1992. A strain of Flavobacterium odoratum isolated from liver hydatid postoperative drainage liquid. Lab. Med., (01):7 (in Chinese).

Andreoni, S., 1986. Isolation of Flavobacterium odoratumfrom human matter. Quad. Sclavo. Diagn., 22(3): 318-329.

Bachmeyer, C., Entressengle, H., Khosrotehrani, K., et al., 2007. Cellulitis due to Myroides odoratimimus in a patient with alcoholic cirrhosis. Clin. Exp. Dermatol., 33: 97-98.

http://dx.doi.org/10.1111/j.1365-2230.2007.02590.x

Benedetti, P., Rassu, M., Pavan, G., et al., 2011. Septic shock, pneumonia, and soft tissue infection due to Myroides odoratimimus: report of a case and review of Myroides infections. Infection, 39(2):161-165. http://dx.doi.org/10.1007/s15010-010-0077-1

Bentley, D., 2006. Whole-genome re-sequencing. Curr. Opin. Genet. Dev., 16(6):545-552.

http://dx.doi.org/10.1016/j.gde.2006.10.009

Burghartz, M., Bunk, B., Spröer, C., et al., 2015. Complete genome sequence of the urethral catheter isolate Myroides sp. A21. Genome Announc., 3(2):e00068-15. http://dx.doi.org/10.1128/genomeA.00068-15

Chen, X.C., Liang, L., Lin, W., 2009. Analysis of elven Myroides sp. isolates from urine culture. Chin. J. Pract. Med. Tech., 16(9):691-692 (in Chinese).
Chi, K.R., 2008. The year of sequencing. Nat. Methods, 5(1): $11-14$. http://dx.doi.org/10.1038/nmeth1154

Cho, S.H., Chae, S.H., Im, W.T., et al., 2011. Myroides marinus sp. nov., a member of the family Flavobacteriaceae, isolated from seawater. Int. J. Syst. Evol. Microbiol., 61(Pt 4):938-947.

http://dx.doi.org/10.1099/ijs.0.024067-0

Crum-Cianflone, N.F., Matson, R.W., Ballon-Landa, G., 2014. Fatal case of necrotizing fasciitis due to Myroides odoratus. Infection, 42(5):931-935. http://dx.doi.org/10.1007/s15010-014-0626-0

Daum, L.T., Fischer, G.W., Sromek, J., et al., 2014. Characterization of multi-drug resistant Mycobacterium tuberculosis from immigrants residing in the USA using Ion Torrent full-gene sequencing. Epidemiol. Infect., 142(6): 1328-1333.

http://dx.doi.org/10.1017/S0950268813002409

Geng, H.Q., Li, X.L., Li, S., 2000. Isolating and identifying a strain of Flavobacterium odoratum in blood and bone marrow. Cent. Plains Med. J., 27(3):52 (in Chinese).

Guo, L., Liu, X.F., 2011. Separation of imipenem-resistant Flavobacterium odoratum from sputum in one case. Lab. Med. Clin., 8(2):175-177 (in Chinese). http://dx.doi.org/10.3969/j.issn.1672-9455.2011.02.023

Holmes, B., Snell, J.J.S., Lapage, S.P., 1977. Revised description, from clinical isolates, of Flavobacterium odoratum Stutzer and Kwaschnina 1929, and designation of the neotype strain. Int. J. Syst. Bacteriol., 27(4):330-336. http://dx.doi.org/10.1099/00207713-27-4-330

Hu, X.L., He, J.Y., Fu, P.M., et al., 2013. Participation of clinical pharmacists in one case of bone tissue infection by multidrug-resistant Flavobacterium odoratum and 
review of the literature. China Pharm., 24(30):2873-2875 (in Chinese).

Huang, H., Yang, Z.L., Wu, X.M., et al., 2012. Complete genome sequence of Acinetobacter baumannii MDR-TJ and insights into its mechanism of antibiotic resistance. $J$. Antimicrob. Chemoth., 67(12):2825-2832. http://dx.doi.org/10.1093/jac/dks327

Huang, Y.K., Yao, Y., Wang, J., et al., 2014. One case of cellulitis complicated with bacteremia caused by multidrugresistant fungus genus. J. Clin. Lab., 32(7):560 (in Chinese). http://dx.doi.org/10.13602/j.cnki.jcls.2014.07.26

Huang, Y.M., Lin, Y., 2003. A case of Flavobacterium odoratum septicemia. Chin. J. Infect. Dis., 21(2):131 (in Chinese).

Hummel, A.S., Hertel, C., Holzapfel, W.H., et al., 2007. Antibiotic resistances of starter and probiotic strains of lactic acid bacteria. Appl. Environ. Microbiol., 73(3):730-739. http://dx.doi.org/10.1128/AEM.02105-06

Jin, Z.C., Xiao, Z.M., 1995. A case of nosocomial infection by Myroides sp. Chin. J. Pract. Intern. Med., 15(3):162 (in Chinese).

Koren, S., Schatz, M.C., Walenz, B.P., et al., 2012. Hybrid error correction and de novo assembly of single-molecule sequencing reads. Nat. Biotechnol., 30(7):693-700. http://dx.doi.org/10.1038/nbt.2280

Ktari, S., Mnif, B., Koubaa, M., et al., 2012. Nosocomial outbreak of Myroides odoratimimus urinary tract infection in a Tunisian hospital. J. Hosp. Infect., 80(1):77-81. http://dx.doi.org/10.1016/j.jhin.2011.09.010

Kuai, S., Huang, L.H., Pei, H., et al., 2011. Imipenem resistance due to class A carbapenemase KPC-2 in a Flavobacterium odoratum isolate. J. Med. Microbiol., 60(Pt 9): 1408-1409. http://dx.doi.org/10.1099/jmm.0.029660-0

Lan, Z.C., Bao, G.J., 2009. The culture, isolation, identification and antimicrobial susceptibility testing of 23 strains of Myroides sp. Chin. Commun. Dr., 11(14):162 (in Chinese). http://dx.doi.org/10.3969/j.issn.1007-614x.2009.14.181

Lee, M.H., Chen, T.L., Lee, Y.T., et al., 2013. Dissemination of multidrug-resistant Acinetobacter baumannii carrying $B l a_{\text {OxA-23 }}$ from hospitals in central Taiwan. J. Microbiol. Immunol. Infect., 46(6):419-424. http://dx.doi.org/10.1016/j.jmii.2012.08.006

Lee, M.J., Jang, S.J., Li, X.M., et al., 2014. Comparison of $r p o B$ gene sequencing, $16 \mathrm{~S}$ rRNA gene sequencing, gyr $B$ multiplex PCR and the VITEK2 system for identification of Acinetobacter clinical isolates. Diagn. Microbiol. Infect. Dis., 78(1):29-34. http://dx.doi.org/10.1016/j.diagmicrobio.2013.07.013

Li, C.Y., Zhao, T.L., 1995. Isolation, identification and drug sensitivity results of 12 strains of Flavobacterium odoratum. Shanghai J. Med. Lab. Sci., 10(12):96 (in Chinese).

Li, H., Li, Y., Xian, X., et al., 2010. Three isolates of Myroides sp. from middle urinary tract. Clin. Focus, 25(13):1125 (in Chinese).
Liu, Z.Y., He, Y., 2001. A case of pulmonary infection caused by Clay Shah Ray Prandlofs real bacteria and Flavobacterium odoratum. Pract. Med. Technol., 8(3):175 (in Chinese)

Macfarlane, D.E., Baum-Thureen, P., Crandon, I., 1985. Flavobacterium odoratum ventriculitis treated with intraventricular cefotaxime. J. Infect., 11(3):233-238. http://dx.doi.org/10.1016/S0163-4453(85)93228-1

Mammeri, H., Bellais, S., Nordmann, P., 2002. Chromosomeencoded $\beta$-lactamases TUS- 1 and MUS-1 from Myroides odoratus and Myroides odoratimimus (formerly Flavobacterium odoratum), new members of the lineage of molecular subclass B1 metalloenzymes. Antimicrob. Agents Chemother., 46(11):3561-3567. http://dx.doi.org/10.1128/AAC.46.11.3561-3567.2002

Maraki, S., Sarchianaki, E., Barbagadakis, S., 2012. Myroides odoratimimus soft tissue infection in an immunocompetent child following a pig bite: case report and literature review. Braz. J. Infect. Dis., 16(4):390-392. http://dx.doi.org/10.1016/j.bjid.2012.06.004

Marathe, N.P., Shetty, S.A., Lanjekar, V.B., et al., 2014. Genome sequencing of multidrug resistant novel Clostridium sp. BL8 reveals its potential for pathogenicity. Gut Pathog., 6(1):30. http://dx.doi.org/10.1186/1757-4749-6-30

Margulies, M., Egholm, M., Altman, W.E., et al., 2005. Genome sequencing in microfabricated high-density picolitre reactors. Nature, 437:376-380. http://dx.doi.org/10.1038/nature03959

Meng, X.X., Xu, X.F., Lang, Q.L., 1999. Flavobacterium and Escherichia coli were isolated from the blood, bile, ascites at the same time. Shanghai Med. Test J., 14(6):372 (in Chinese)

Ravindran, C., Varatharajan, G.R., Raju, R., et al., 2015. Infection and pathogenecity of Myroides odoratimimus (NIOCR-12) isolated from the gut of grey mullet (Mugil cephalus (Linnaeus, 1758)). Microb. Pathog., 88:22-28. http://dx.doi.org/10.1016/j.micpath.2015.08.001

Rolain, J.M., Diene, S.M., Kempf, M., et al., 2013. Real-time sequencing to decipher the molecular mechanism of resistance of a clinical pan-drug-resistant baumannii isolate from Marseille, France. Antimicrob. Agents Chemother., 57(1):592-596. http://dx.doi.org/10.1128/AAC.01314-12

Rothberg, J.M., Hinz, W., Rearick, T.M., et al., 2011. An integrated semiconductor device enabling non-optical genome sequencing. Nature, 475(7356):348-352. http://dx.doi.org/10.1038/nature10242

Schröttner, P., Rudolph, W.W., Eing, B.R., et al., 2014. Comparison of VITEK2, MALDI-TOF MS, and 16S rDNA sequencing for identification of Myroides odoratus and Myroides odoratimimus. Diagn. Microbiol. Infect. Dis., 79(2):155-159. http://dx.doi.org/10.1016/j.diagmicrobio.2014.02.002

Shi, W.F., Zhou, K.Q., 1993. Isolation of a strain of Flavobacterium odoratum from cerebrospinal fluid. Shanghai $J$. 
Med. Lab. Sci., 8(4):211 (in Chinese).

Snitkin, E.S., Zelazny, A.M., Gupta, J., et al., 2013. Genomic insights into the fate of colistin resistance and Acinetobacter baumannii during patient treatment. Genome Res., 23(7):1155-1162.

http://dx.doi.org/10.1101/gr.154328.112

Song, C.G., 2005. One case of severe iatrogenic pulmonary infection caused by Flavobacterium odoratum. Chin. J. Hosp. Infect., 15(10):1141 (in Chinese).

Song, K.Y., Zhang, H.W., Zhang, X.Y., 1995. A case of chewing muscle abscess caused by Flavobacterium odoratum. Shanghai Med. Test J., 10(4):245 (in Chinese).

Spanik, S., Trupl, J., Krcmery, V., 1998. Nosocomial catheterassociated Flavobacterium odoraturn bacteraemia in cancer patients. J. Med. Microbiol., 47(2):183. http://dx.doi.org/10.1099/00222615-47-2-183

Stutzer, M., Kwaschnina, A., 1929. Aussaaten aus den Fäzes des Menschen gelbe Kolonien bildende Bakterien (Gattung Flavobacterium u.a.). Zentralbl. Bakteriol. Parasitenkd. Infektionskr. Hyg. Abt. 1 Orig., 113:219-225 (in German).

Suganthi, R., Priya, T.S., Saranya, A., et al., 2013. Relationship between plasmid occurrence and antibiotic resistance in Myroides odoratimimus SKS05-GRD isolated from raw chicken meat. World J. Microbiol. Biotechnol., 29(6): 983-990. http://dx.doi.org/10.1007/s11274-013-1257-9

Sun, X.H., Zhang, C.L., 2006. A strain of Flavobacterium odoratum isolated from wounds in one case of traumatic patient. Chin. Commun. Dr., 12(8):82 (in Chinese).

Tan, S.Y., Chua, S.L., Liu, Y., et al., 2013. Comparative genomic analysis of rapid evolution of an extreme-drug-resistant baumannii clone. Genome Biol. Evol., 5(5):807-818. http://dx.doi.org/10.1093/gbe/evt047

Tatusova, T., Ciufo, S., Federhen, S., et al., 2015. Update on RefSeq microbial genomes resources. Nucleic Acids Res., 43(D1):D599-D605. http://dx.doi.org/10.1093/nar/gku1062

Tian, G.Z., Wang, X.L., 2010. Advances in the study of Flavobacterium indologenes. J. Pathog. Biol., 5(2):134-136 (in Chinese). http://dx.doi.org/10.13350/j.cjpb.2010.02.018

Vancanneyt, M., Segers, P., Torck, U., et al., 1996. Reclassification of Flavobacterium odoratum (Stutzer 1929) strains to a new genus, Myroides, as Myroides odoratus comb. nov. and Myroides odoratimimus sp. nov. Int. J. Syst. Bacteriol., 46(4):926-932. http://dx.doi.org/10.1099/00207713-46-4-926

Wang, Y.L., Su, F.M., 1992. A case of neonatal septicemia caused by Flavobacterium odoratum. Lab. Med., (4):248 (in Chinese).

Wu, G.M., 1998. Flavobacterium odoratum was detected from a burn patient blood and many other parts. Shanghai J. Med. Lab. Sci., 13(1):34 (in Chinese).
Wuer, G.L., Aisi, K.E., Gu, H.E., 2002. One cases Flavobacterium odoratum isolated from urine separation. J. Clin. Lab., 20(1):44 (in Chinese).

Yağci, A., Cerikçioğlu, N., Kaufmann, M.E., et al., 2000. Molecular typing of Myroides odoratimimus (Flavobacterium odoratum) urinary tract infections in a Turkish hospital. Eur. J. Clin. Microbiol. Infect. Dis., 19(9):731-732. http://dx.doi.org/10.1007/s100960070001

Yan, S., Zhao, N., Zhang, X.H., 2012. Myroides phaeus sp. nov., isolated from human saliva, and emended descriptions of the genus Myroides and the species Myroides profundi Zhang et al. 2009 and Myroides marinus Cho et al. 2011. Int. J. Syst. Evol. Microbiol., 62(Pt 4):770-775. http://dx.doi.org/10.1099/ijs.0.029215-0

Yang, J.H., Wang, L.Y., 2001. A case of Flavobacterium odoratum wound infection. Pract. Med. Technol., 8(7): 542 (in Chinese).

Yoon, J., Maneerat, S., Kawai, F., et al., 2006. Myroides pelagicus sp. nov., isolated from seawater in Thailand. Int. J. Syst. Evol. Microbiol., 56(Pt 8):1917-1920. http://dx.doi.org/10.1099/ijs.0.64336-0

Zhang, B.C., Zhang, J.T., 1996. Neonatal sepsis associated with meningitis and strain identification of Flavobacterium odoratum. Tianjin Med., 24(3):181-182 (in Chinese).

Zhang, X.Y., Zhang, Y.J., Chen, X.L., et al., 2008. Myroides profundi sp. nov., isolated from deep-sea sediment of the southern Okinawa Trough. FEMS Microbiol. Lett., 287(1): 108-112. http://dx.doi.org/10.1111/j.1574-6968.2008.01299.x

Zhang, Z.D., He, L.Y., Huang, Z., et al., 2014. Myroides xuanwuensis sp. nov., a mineral-weathering bacterium isolated from forest soil. Int. J. Syst. Evol. Microbiol., 64(Pt 2):621-624. http://dx.doi.org/10.1099/ijs.0.056739-0

Zhao, S.Q., 2000. One case of children with Flavobacterium odoratum septicemia. New Med., 31(8):482-483 (in Chinese).

\section{中文概要}

\section{题 目: 芳香黄杆菌的耐药机制}

概 要: 芳香黄杆菌广泛耐药, 一旦感染很难治愈。其耐 药机制尚不清楚, 需要进一步研究。本文对国内 外的芳香黄杆菌病例进行全面总结, 分析该细菌 的抗生素耐药情况、耐药机制、病人预后、医院 内爆发感染和流行的风险, 以及目前实验室应用 的诊断方法, 指出全基因组测序应用于阐明其耐 药机制的可行性和迫切性。

关键词: 芳香黄杆菌; 抗生素耐药; 鉴定方法; 16S rRNA 基因测序; 下一代测序 